CARNETS DE Carnets de géographes

GÉOGRAPHES.

$8 \mid 2015$

Géographie(s) de la lenteur

\title{
Inégalités et (in)justices spatiales à Maputo
}

Pratiques des services urbains (Mozambique)

\section{Karine Ginisty}

\section{(2) OpenEdition}

Journals

Édition électronique

URL : http://journals.openedition.org/cdg/330

DOI : $10.4000 /$ cdg.330

ISSN : 2107-7266

Éditeur

UMR 245 - CESSMA

Référence électronique

Karine Ginisty, «Inégalités et (in)justices spatiales à Maputo », Carnets de géographes [En ligne], 8 |

2015, mis en ligne le 01 septembre 2015, consulté le 24 septembre 2020. URL : http://

journals.openedition.org/cdg/330 ; DOI : https://doi.org/10.4000/cdg.330

Ce document a été généré automatiquement le 24 septembre 2020.

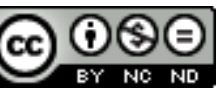

La revue Carnets de géographes est mise à disposition selon les termes de la Licence Creative Commons Attribution - Pas d'Utilisation Commerciale - Pas de Modification 4.0 International. 


\section{Inégalités et (in)justices spatiales à Maputo}

Pratiques des services urbains (Mozambique)

\section{Karine Ginisty}

1 Cette thèse de doctorat porte sur la justice et l'injustice spatiales à Maputo, avec pour objet de recherche les inégalités d'accès aux services urbains (eau potable, collecte des déchets ménagers). Construite sur l'hypothèse d'un lien entre le degré de spatialisation des inégalités d'accès aux services urbains et la formation de sentiments d'injustice, la thèse se détourne toutefois d'un projet normatif sur ce que serait le juste et l'injuste par et dans la production de l'espace. L'enjeu principal de cette thèse est la construction d'un questionnement sur la dimension spatiale de la justice sociale à partir des catégories politiques, de l'action publique et citadines du juste et de l'injuste à Maputo.

2 La thèse adopte une démarche postcoloniale ; elle positionne l'analyse de la justice et l'injustice en ville sous l'angle des acteurs qui produisent, vivent et perçoivent les inégalités d'accès aux services urbains à Maputo. Ce positionnement signifie une méthodologique qui accorde une place centrale à l'ethnographie, à la fois comme matériau et résultat de la recherche. Ainsi, le terrain a été centré sur les expériences quotidiennes des inégalités d'accès à deux services urbains de 200 citadins répartis dans cinq Bairros périphériques de Maputo entre 2008 et 2010. La focale sur les pratiques d'approvisionnement en eau et de la gestion des déchets ménagers visait à explorer les raisons du silence des citadins face aux injustices vécues, même lorsque le maintien en ville est compromis. La thèse entreprend donc par cette voie de déconstruire les processus de catégorisation du juste et de l'injuste qui prennent forme localement, considérant aussi bien le poids des héritages urbains, que celui des trajectoires citadines ou de la quotidienneté. Si l'échelle de l'espace vécu a été privilégiée pour construire la problématique et la méthodologie afférente, l'analyse des moments durant lesquels les acteurs énoncent l'injustice spatiale ou s'y refusent s'inscrit à une échelle plus vaste. La catégorisation de l'injustice dans les registres du politique, et en particulier de la critique politique, s'accompagne d'une circonscription de l'expression 
du juste et de l'injuste à la sphère de l'intime, ancrée dans les cercles familiaux et amicaux. L'absence de traduction des sentiments d'injustice dans des registres d'action collective a alors soutenu une approche multi-scalaire du politique. Le politique fut aussi bien approché sous l'angle des structures de pouvoir municipales, des référentiels et des pratiques de pouvoir qui y prévalaient, que des imaginaires politiques et de la quotidienneté du politique à l'échelle locale.

3 Les apports de cette thèse résident dans la manière de saisir la place du politique dans la production de la justice et de l'injustice spatiales, à différents moments et échelles de la production de l'espace urbain. Constituée de cinq chapitres, la thèse s'ouvre à l'indépendance, lorsque Lourenço Marques devint Maputo, une ville socialiste et postcoloniale. Le premier chapitre "Écrire la justice spatiale de Lourenço Marques à Maputo » revient sur la construction d'un modèle de ville plus juste par l'Etat-Frelimo - Front de Libération du Mozambique - et les enjeux politiques de sa mise en scène publique, puis de la fabrique de la mémoire collective et son oubli. Il renseigne sur le lien étroit entre la «lutte juste» et l'autoritarisme du Frelimo, posant en filigrane la question des héritages de la ville socialiste tout du long de la thèse.

Ensuite, le chapitre "Décider de la justice spatiale dans les arènes municipales » resitue la problématique de la justice spatiale dans les années 2000, dans une capitale désormais vitrine internationale du développement et de la démocratie au Mozambique. Dans les coulisses de l'action publique, ce chapitre explore les raisons du silence public des politiques, des agents publics et des coopérants sur la justice spatiale promue, alors même qu'une action publique est développée pour réduire les inégalités urbaines. La technicisation du problème public de la gestion des déchets dans l'espace public est ainsi étudiée au prisme des tensions partisanes qui se forment autour des mutations de l'action publique et de la politisation des inégalités urbaines qui l'accompagne, deux décennies après l'abandon de la doctrine socialiste.

5 Dans cette thèse, les processus de décision de l'action publique sont analysés séparément de la production de l'espace en elle-même. Les dispositifs et les arrangements spatiaux promus dans le cadre d'un Partenariat Public-Privé (eau potable) et d'un projet de coopération à l'échelle municipale (déchets) sont présentés dans le chapitre "Traduire la justice spatiale dans les quartiers périphériques». Ce dernier s'attache à cartographier la restructuration des inégalités spatiales d'accès aux services urbains et pose le décor d'une fragmentation urbaine. Dans ce chapitre, Maputo, ville duale, évolue vers une configuration tripartite de ses ressources urbaines, distinguant le centre, les quartiers péricentraux et les quartiers ultra-périphériques.

6 La reconfiguration des inégalités urbaines constitue un moment privilégié pour étudier le rapport au juste et à l'injuste des citadins à travers l'expérience quotidienne des services urbains. La thèse se poursuit donc sur le chapitre «Vivre les inégalités, ressentir l'injustice", qui décrit la quotidienneté des services urbains dans les quartiers périphériques. La praxis sociale du service de l'eau potable est confrontée aux catégorisations citadines du juste et de l'injuste. Ce chapitre démontre alors que le degré d'inégalité d'accès au service de l'eau potable n'intervient pas dans la formation des sentiments d'injustice et leur spatialisation. Ressentir une injustice dans les scènes de la vie quotidienne interroge plutôt les usages socio-politiques du mot injustice (sens du dire) et les espaces d'expression individuels et collectifs accessibles (lieu du dit). Les sentiments d'injustice sont pourtant présents dans les quartiers périphériques de 
Maputo, mais ils sont vécus comme une expérience intime, indicible dans l'espace public.

7 Le dernier chapitre, "Dire et taire l'injustice dans les enclaves autoritaires de la ville", s'attache alors revenir sur l'expérience politique des espaces publics dont peuvent s'emparer les citadins à l'échelle locale. Le contrôle politique de ces espaces révèle la permanence de pratiques de pouvoir autoritaires, construites sous le régime de l'EtatParti. Ces pratiques éclairent le détournement des citadins de l'expression collective des injustices vécues. La confusion des fonctions publiques et partidaires à l'échelle des Quarteirao et des Bairros, les pratiques de verrouillage du débat public et la permanence $\mathrm{du}$ personnel politique local concourent à la formation d'enclaves de pouvoir autoritaire. La thèse s'achève alors sur l'expérience politique des cadres locaux du Frelimo et leur rapport aux injustices spatiales.

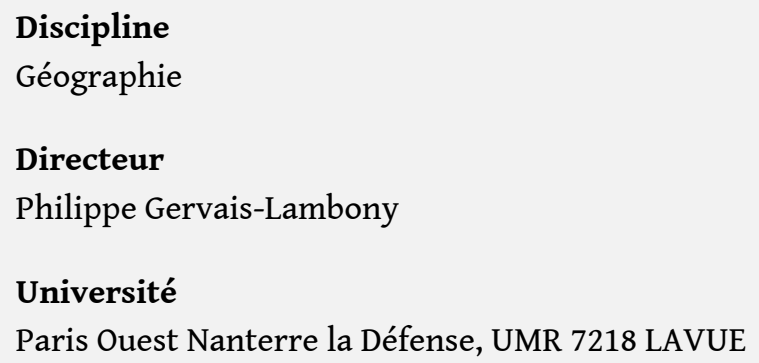

INDEX

Thèmes : Carnets de soutenances 